\title{
Study on the Multi-Component Quantitation of Cortex Moutan and Its Intestinal Absorption Characteristics
}

\author{
Mei Ge, Xihong Hou, Shimeng Li, Kunpeng Liu, Ziyi Han, Siyu Hao, \\ Zhongliang Zeng*, Yingchun Zhang* \\ College of Pharmaceutical Sciences and Chinese Medicine, Southwest University, Chongqing, China \\ Email: *zzl1998@163.com, *yczhang1203@swu.edu.cn
}

How to cite this paper: Ge, M., Hou, X.H., Li, S.M., Liu, K.P., Han, Z.Y., Hao, S.Y., Zeng, Z.L. and Zhang, Y.C. (2019) Study on the Multi-Component Quantitation of Cortex Moutan and Its Intestinal Absorption Characteristics. Pharmacology \& Pharmacy, 10, 146-158.

https://doi.org/10.4236/pp.2019.103013

Received: February 26, 2019

Accepted: March 22, 2019

Published: March 25, 2019

Copyright $\odot 2019$ by author(s) and Scientific Research Publishing Inc. This work is licensed under the Creative Commons Attribution International License (CC BY 4.0). http://creativecommons.org/licenses/by/4.0/

\section{Open Access}

\begin{abstract}
Cortex Moutan (Paeonia suffruticosa Andr.) is a common traditional Chinese medicine and has been widely used in clinic for 2000 years in China. As sources for this crude drug are always mixed with other species, many cultivars on herbal market may lead to quality instability. Multi-component quantitative analysis is an efficient method to reflect chemical profiles of herb medicine and is always taken as the main method for quality evaluation. So, the aim of this work is to develop analytical method to quantify paeonol, paeoniflorin, gallic acid, oxypaeoniflorin, benzoylpaeoniflorin and paeonolide in Cortex Moutan (CM) to evaluate the chemical qualities of CM from different species or cultivars. Besides, we also study the intestinal absorption characteristics of paeonol and paeoniflorin for further pharmacological evaluation. In the present study, all of the standard markers were performed on an Ecosil $\mathrm{C}_{18}(250 \mathrm{~mm} \times 4.6 \mathrm{~mm}, 5 \mu \mathrm{M}$, Lubex Co., Guangzhou, China) with linear gradient elution of $0.2 \%$ formic acid water and acetonitrile. The proposed method was applied to analyze 50 batches of samples with acceptable linearity $\left(\mathrm{R}^{2}, 0.9995-0.9999\right)$, precisions ( $\left.\mathrm{RSD}, 0.47 \%-2.08 \%\right)$, repeatability (RSD, $039 \%-2.63 \%$ ), stability (RSD, $0.52 \%-2.45 \%$ ), and recovery (RSD, $0.72 \%-3.03 \%$ ) of the six compounds. Furthermore, the Hierarchical Cluster Analysis was applied to classify the 50 samples based on contents of the six compound markers. The results obtained from multi-component quantification of CM clearly indicated that CM originated from P. suffruticosa and $P$. ostii presented different chemical properties, and that samples from the two materials could be gathered into one branch, respectively, while CM sourced from cultivars of $P$. suffruticosa showed great variety on chemical quality. The results from Hierarchical Cluster Analysis implied that the established method could be used as a powerful tool for the quality evaluation of CM.
\end{abstract}


The intestinal absorption study indicated that the intestinal absorption activities for paeoniflorin and paeonol showed an increasing absorption with time. Paeonol had lower absorption rate $(6.69 \%$ - $15.93 \%)$ than that of paeoniflorin $(19.0 \%-30.70 \%)$. As a result, the established method is suitable for the quality evaluation of CM. The results of intestinal absorption characteristics of paeonol and paeoniflorin offer an insight for pharmacological evaluation and clinical efficacy research of CM.

\section{Keywords}

Cortex Moutan, Paeonol, Paeoniflorin, Everted Intestinal Sac, HPLC-MS

\section{Introduction}

Paeonia suffruticosa Andr. is a famous ornamental plant in China and is widely cultivated throughout the world [1]. Cortex Moutan (CM), from the dried root bark of Paeonia suffruticosa Andr. [2], has been used as a traditional Chinese medicine with treatment of blood related disorders for 2000 years [3]. CM is also widely used in Chinese Patent Medicines as one sub-material, such as in famous LiuweiDihuang preparations, GuizhiFuling tablet and Rhubarb Peony Decoction [4] [5] [6]. According to Chinese Pharmacopoeia, CM should originate from Paeonia Suffruticosa Andr. [2], while another CM called "Fengdanpi", which is derived from $P$. ostii T. Hong et J.X. Zhang, mainly growing in Anhui province, is also widely circulated on herbal market and used as a major source of CM in clinic [7].

As a common traditional Chinese medicine, CM is believed to have effects of clearing away heat and cooling blood, activating blood circulation to dissipate blood stasis [8]. At present, seven classes of compounds have been isolated from CM: phenols, monoterpenes, monoterpene glycosides, flavonoids, tannins, triterpenoids and others [9] [10] [11] [12]. Phenols and monoterpene glycosides are the predominant active ingredients [13] [14] [15] [16]. Nevertheless, the therapeutic properties of CM always correlate with its sources and geographical origins [17]. Besides, some CMs originated from cultivars may circulate on the market. Thus, this circumstance may result in quality instability and further affect clinical efficacy of CM [13]. So, it is urgent to develop an effective method to evaluate quality of CM.

To date, only one or two bioactive components such as paeonol or paeoniflorin have been chosen as chemical markers for quality assessment of CM [18] [19], which may not reflect overall chemical quality of CM. High performance liquid chromatography coupled with Mass spectrum (HPLC-MS) is the main regular technique for the qualitative or quantitative analysis in herbal medicine [20] [21]. In the previous study, we had established a HPLC fingerprint of CM and identified the most components from the common peaks by Mass spectrum technique. Among the identified common ingredients, paeonol, paeoniflorin, 
gallic acid, oxypaeoniflorin, benzoylpaeoniflorin and paeonolide showed higher contents in the CM fingerprint [22]. So, the present study will focus on the quantification of the six compounds (Figure 1) in CM from different sources and geographical origins.

For herbal medicine, only the absorbed constituents may produce possible pharmacological effects. However, crude herbal extracts are often directly added into cells or organs culture system in vitro in some studies, without considering of absorption process, as not all the components could be absorbed or fully absorbed. The false positive results or the false negative results may occur easily. Furthermore, the real pharmacological effects of crude drugs are also a reflection of its quality. Thus, intestinal absorption characteristics of crude herbal drugs are needed to consider. As paeonol and paeoniflorin are the main components in $\mathrm{CM}$, it is necessary to study their intestinal absorption characteristics for clinical efficacy evaluation of CM. As everted intestinal sac test is a simple and effective method to reflect absorption activities of components from crude drugs [23] [24], we have tested the absorption activities of paeonol and paeoniflorin in jejunum and ileum at different absorption time.

\section{Materials and Methods}

\subsection{Chemical Reagents and Crude Drug Materials}

Six standard compounds of gallic acid, oxypaeoniflorin, paeonolide, paeoniflorin, benzoylpaeoniflorin and paeonol were purchased from the National Institute for the Control of Pharmaceutical and Biological Products (Beijing, China). The purity of each standard compound was above $98 \%$ and suitable for HPLC analysis. HPLC grade Acetonitrile was purchased from Tedia Company Inc. (Fairfield, OH, USA). Formic acid of HPLC-grade was purchased from Chongqing Chuandong Chemical co., Ltd. (Chongqing, China). Methanol for extraction was purchased from Chendu Jinshan Chemical Reagent co., Ltd. (Chengdu, China). Water was purified using an ultra-pure water machine from Sichuan Youpu Ultra-pure Technology Co., Ltd. (Sichuan, China).

Fifty batches of CM (S1-S50) were collected from three main production regions in China. Of these, S1-S24 originated from Paeonia suffruticosa Andr. were<smiles>O=C(O)c1cc(O)c(O)c(O)c1</smiles>

(1)<smiles>COc1ccc(C(C)=O)c(O)c1</smiles>

(2)

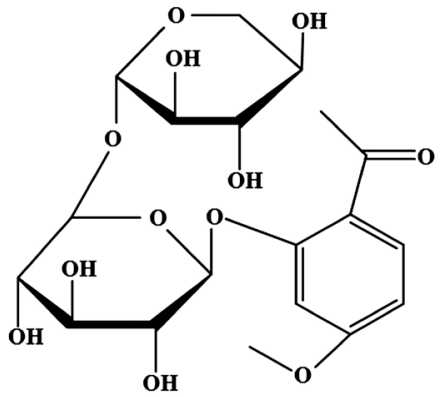

(3)

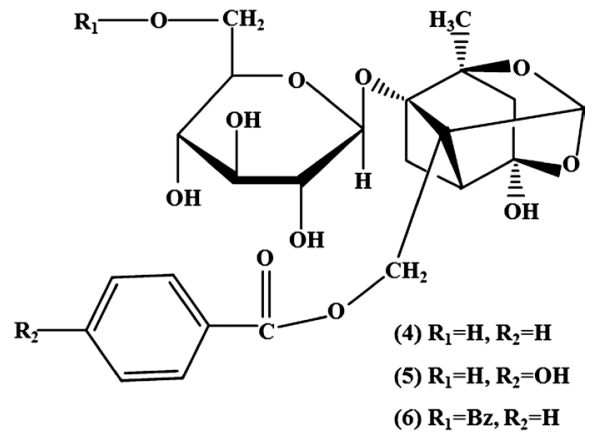

Figure 1. The chemical structures of the six components: (1) gallic acid; (2) paeonol; (3) paeonolide; (4) paeoniflorin; (5) oxypaeoniflorin; (6) benzoylpaeoniflorin. 
collected from Chongqing, China. While S25-S40 sourced from P. ostii T. Hong et J.X. Zhang, in which S25-S32 were harvested from Chongqing, China, and S33-S40 were from Anhui province of China. Another ten individuals (S41-S50) were grown in Henan province and originated from ten cultivars of Paeonia suffruticosa Andr. All the samples were authenticated with morphological and histological methods by associate professor Hanru Liu from the College of Pharmaceutical Sciences and Chinese Medicine, Southwest University. Voucher specimens were deposited at College of Pharmaceutical Sciences and Chinese Medicine, Southwest University. Each sample was cored and dried in $50^{\circ} \mathrm{C}$ for HPLC analysis.

\subsection{Preparation of Standard and Sample Solution}

A mixed stock standard solution containing gallic acid, oxypaeoniflorin, paeonolide, paeoniflorin, benzoylpaeoniflorin and paeonol was prepared by accurately weighing appropriate amounts of the six reference compounds and dissolving in $70 \%$ methanol. All the standard stock and working solutions were stored at $4^{\circ} \mathrm{C}$.

All the materials were dried to constant weight, then pulverized and sifted through a 40 mesh sieve. Powdered sample $(0.5 \mathrm{~g})$ was weighed accurately, and all samples were ultrasonically extracted using $50 \mathrm{~mL}$ 70\% methanol for $30 \mathrm{~min}$ at room temperature, and then settled to the volume of $50 \mathrm{~mL}$. The extracted solution was centrifuged at $12,000 \mathrm{rpm}$ for $5 \mathrm{~min}$, and the supernatant was filtered through a $0.22 \mu \mathrm{M}$ nylon membrane filter. The filtrates were analyzed directly by HPLC-MS.

$\mathrm{CM}$ extract was prepared with $70 \%$ ethanol under reflux for $2 \mathrm{~h}$. The extraction process was repeated twice. The extract was concentrated under reduced pressure using the rotary evaporator, and followed by dilution with Tyrode buffer to the concentration of $0.1 \mathrm{~g} / \mathrm{mL}$.

\subsection{Experimental Animals}

Adult male Sprague-Dawley rats weighing (220 - $250 \mathrm{~g}$ ) were obtained from Chongqing Academy of Chinese Materia Medica, China. The rats were used for the everted gut sac experiment. The animal welfare and experimental procedures comply with the Guide for the Care and Use of Laboratory Animals (National Research Council of the USA, 1996) and related ethical regulations of Southwest University.

\subsection{Instrumentation and Analytical Conditions}

The HPLC analyses were performed using liquid chromatography system (Shimadzu Co., Japan) coupled with a mass spectrometer equipped with an electrospray ion source (ESI-MS) (Shimadzu Co., Japan). Samples were separated on an Ecosil $\mathrm{C}_{18}(250 \mathrm{~mm} \times 4.6 \mathrm{~mm}, 5 \mu \mathrm{M}$, Lubex Co., Guangzhou, China). The linear gradient conditions were solvent $\mathrm{A}$ of $0.2 \%$ formic acid in deionized water and 
solvent B of acetonitrile using a gradient program of 0 - $3 \mathrm{~min}, 10 \%-11.8 \% \mathrm{~B} ; 3$ - $5 \mathrm{~min}, 11.8 \%$ - 12\% B; 5 - $7 \mathrm{~min}, 12 \%$ - 12.1\% B; 7 - $9 \mathrm{~min}, 12.1 \%$ - 12.2\% B; 9 $10 \min , 12.2 \%$ - $12.3 \% \mathrm{~B} ; 10$ - $15 \min , 12.3 \%$ - 13\% B; 15 - $18 \mathrm{~min}, 13 \%$ - 15\% B; 18 - $21 \mathrm{~min}, 15 \%$ - 15.8\% B; 21 - $26 \mathrm{~min}, 15.8 \%$ - 74\% B; 26 - $30 \mathrm{~min}, 74 \%$ - 90\% B; $30-35 \mathrm{~min}, 90 \%-90 \%$ B. Elution was performed with a solvent at the flow rate of $1.0 \mathrm{~mL} / \mathrm{min}$, and the injection volume was $10 \mu \mathrm{L}$ for analysis. Ultraviolet spectra were monitored at $230 \mathrm{~nm}$ for acquiring chromatograms of paeoniflorin and benzoylpaeoniflorin, $274 \mathrm{~nm}$ for acquiring chromatograms of gallic acid, paeonol, paeonolide and oxypaeoniflorin. ESI-MS spectra were acquired in negative ion mode to identify the six components. The conditions of MS analysis were designed as follows: the electrospray capillary voltage at $4-10 \mathrm{~V}$; nitrogen was used as a drying gas at the flow rate of $15 \mathrm{~L} / \mathrm{min}$ with a temperature of $350^{\circ} \mathrm{C}$. The full-scan range was from 100 to $1200 \mathrm{~m} / \mathrm{z}$ (Figure 2).

\subsection{Assay Validation}

The quantitative analysis of six standard compounds was validated under the above-described optimized conditions in terms of linearity, precision, stability, repeatability and recovery. A series of standard solutions for constructing working standard curves were prepared by diluting the mixed stock standard solution $1,2,4,8,16,32,64$ times with 70\% methanol, and a calibration curve was established by plotting peak areas (y axis) versus concentrations ( $\mathrm{x}$ axis). The limit of detection (LOD) and lower limits of quantification (LOQ) of the six standard compounds were determined by continuously diluting the standard solution until the $\mathrm{S} / \mathrm{N}$ (signal to noise) ratios reached around 3 and 10, respectively. A mixed standard solution was analyzed for six replicates within the same day for evaluating intra-day precision, and on once for 12 hours at intervals for three consecutive days for evaluating inter-day precision. Repeatability was evaluated by analysis of six samples (S19) which were prepared with the same preparation procedure and processed in parallel as described above. The extract of S19 was analyzed at $0,2,4,8,12,16$ and 24 hour at room temperature for evaluating the

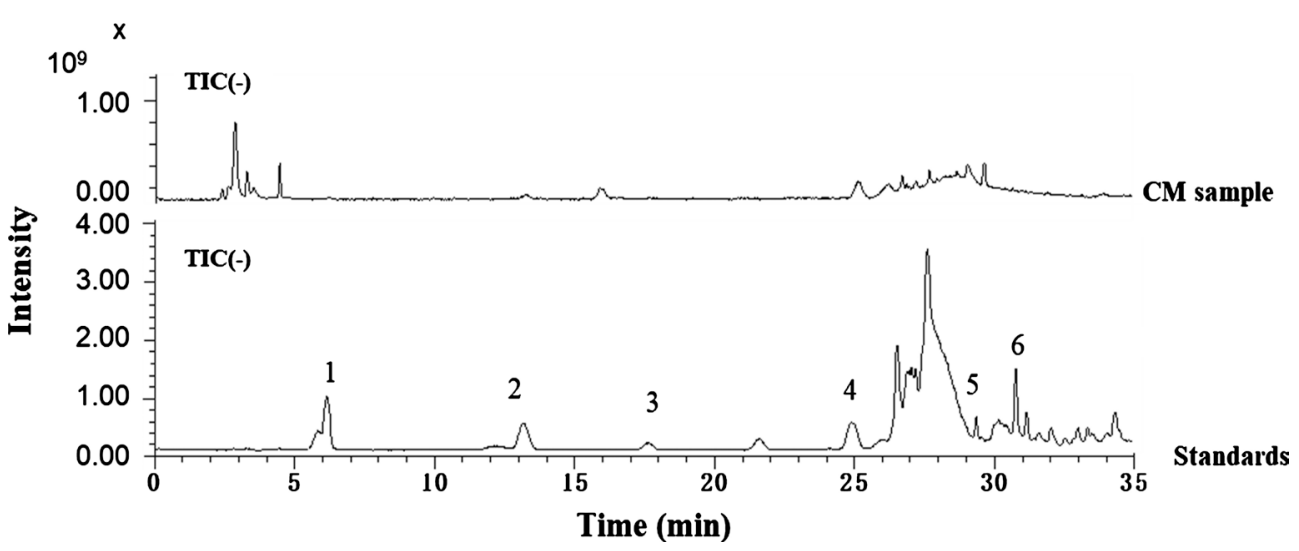

Figure 2. Total ion chromatogram (TIC) of six standards and CM sample. The numbers noted in the spectrum represent the standards: 1-gallic acid; 2-oxypaeoniflorin; 3-paeonolide; 4-paeoniflorin; 5-paeonol; 6-benzoylpaeoniflorin. 
stability. The recovery test was conducted to assess the accuracy of the method by spiking the sample (S19) with different levels of known contents of reference compounds in the sample, so that the samples contain $80 \%, 100 \%$ and $120 \%$ of gallic acid, oxypaeoniflorin, paeonolide, paeoniflorin, benzoylpaeoniflorin and paeonol, respectively.

The injection volume of sample solution and mixed standard solution was 10 $\mu \mathrm{L}$, and the contents of gallic acid, oxypaeoniflorin, paeonolide, paeoniflorin, benzoylpaeoniflorin and paeonol ( $\mathrm{mg} / \mathrm{g}$ ) was calculated according to the external standard method.

\subsection{The Intestinal Absorbed Solution Preparation}

Rats were fasted for $12 \mathrm{~h}$ before the experiment. Under anesthesia, the intestine of each rat was rapidly removed, washed with ice-cold Tyrode buffer solution $\left(\mathrm{mmol} / \mathrm{L}, \mathrm{NaCl}\right.$ 136.75, $\mathrm{KCl} 3.76, \mathrm{NaHCO}_{3}$ 11.90, $\mathrm{NaH}_{2} \mathrm{PO}_{4}$ 0.42, $\mathrm{MgCl}_{2}$ 1.05, $\mathrm{CaCl}_{2} 1.80$, glucose 5.56, $\mathrm{pH}$ 7.4). Jejunum and ileum parts were isolated and cut into $12 \mathrm{~cm}$ segments in length, respectively. Each segment was everted and ligated at both ends to form a sac, and then filled with the Tyrode buffer. The filled sac was incubated in thermal smooth muscle experimental system (TME, Chengdu, China) with Tyrode buffer for $5 \mathrm{~min}$ to guarantee the equilibration, and then the buffer inside the sac was exchanged with the extract solution. During the incubation period, the solution was maintained at $37^{\circ} \mathrm{C}$ and continuously aerated with fresh air. After different absorption time (0.5, 1.0, 1.5, $2.0 \mathrm{~h})$, the sacs were removed and blotted dry with gauze, and the serosal side solutions containing absorbed constituents were drained into small tubes. The intestinal absorbed solutions of CM and blank intestinal absorbed solution were dried by $\mathrm{N}_{2}$ and then ultrasonically extracted with $70 \%$ ethanol for $30 \mathrm{~min}$. These samples were filtered for further analysis.

\section{Results and Discussion}

\subsection{Method Validation and Linearity of Calibration}

The external standard method was established to obtain regression. The calculated results are shown in Table 1, the correlation coefficient values $r \geq 0.9995$,

Table 1. Regression data, linear range and the LOD and LOQ of the developed method.

\begin{tabular}{cccccc}
\hline $\begin{array}{c}\text { Reference } \\
\text { standards }\end{array}$ & Calibration curve & $\mathbf{R}^{2}$ & $\begin{array}{c}\text { Linear range } \\
(\mu \mathrm{g} / \mathrm{mL})\end{array}$ & $\begin{array}{c}\text { LOD } \\
(\mu \mathrm{g} / \mathrm{mL})\end{array}$ & $\begin{array}{c}\text { LOQ } \\
(\mu \mathrm{g} / \mathrm{mL})\end{array}$ \\
\hline Gallic acid & $y=6.9 \times 10^{7} x-16,518$ & 0.9999 & $1.973-126.3$ & 0.009 & 0.029 \\
Oxypaeoniflorin & $y=1.5 \times 10^{7} x-14,298$ & 0.9995 & $3.109-199.0$ & 0.054 & 0.180 \\
Paeonolide & $y=2.8 \times 10^{7} x-4161$ & 0.9999 & $0.752-96.29$ & 0.047 & 0.157 \\
Paeoniflorin & $y=1.1 \times 10^{7} x+41,343$ & 0.9999 & $12.27-392.7$ & 1.319 & 4.398 \\
Benzoylpaeoniflorin & $y=5.2 \times 10^{7} x-34,993$ & 0.9999 & $1.409-90.14$ & 0.204 & 0.680 \\
Paeonol & $y=7.0 \times 10^{7} x-57,901$ & 0.9998 & $4.155-265.9$ & 0.004 & 0.013 \\
\hline
\end{tabular}

LOD refers to the limit of detection, $\mathrm{S} / \mathrm{N}=3$; LOQ refers to the limit of quantification, $\mathrm{S} / \mathrm{N}=10$. 
indicating that the concentrations and their peak areas of all standard compounds had a good linear relationship within the relatively wide test ranges. The Limits of Detection (LOD) was in the range of $0.004-1.319 \mu \mathrm{g} / \mathrm{mL}$. The Limits of Quantification (LOQ) was in the range of $0.013-4.398 \mu \mathrm{g} / \mathrm{mL}$.

The precision of the method was validated by the determination of intra- and inter-day variances. For precision study, the Relative Standard Deviations (RSD) of the mean content for each compound was calculated and ranged from $0.40 \%$ to $2.20 \%$ for intra- and inter-day precisions. The RSD values of all compounds for repeatability study were all less than $2.63 \%$, which indicated good reproducibility of the developed method. As for stability evaluation, the RSD values of the mean content were not more than $2.45 \%$, which indicated that the sample solution was found to be stable. The mean recovery of the method was in the range of $98.05 \%-100.22 \%$, with RSD of less than $3.03 \%$. The results of the recovery assays showed that the method was thus acceptable. All results are listed in Table 2.

\subsection{Quantitative Analysis of Six Compounds}

The developed analytical method was subsequently applied to analysis of six components in 50 individuals of CM. The contents of six constituents with the mean values of three replicate injections were summarized in Table 3 . All the six compounds could be detected in samples and paeoniflorin and paeonol were the main components, which accounted for $60 \%$ - $90 \%$ amounts of all the detected compounds. Besides, samples collected from different areas showed great variety.

\subsection{Quality Assessment of CM by Hierarchical Cluster Analysis}

To evaluate variation of the CM, Hierarchical Cluster Analysis (HCA) was performed based on the chemical characteristics of samples. HCA of 50 samples were established by SPSS software (SPSS 13.0 for Windows, SPSS Inc., USA), in which a method called average linkage between groups was employed and the six markers were selected as the measurement. As shown in Figure 3, two clusters

Table 2. Precision, repeatability, stability and recovery results for the assay of six standards.

\begin{tabular}{|c|c|c|c|c|c|c|c|c|c|c|}
\hline \multirow{3}{*}{ Reference standards } & \multicolumn{4}{|c|}{ Precision $(n=6)$} & \multirow{2}{*}{\multicolumn{2}{|c|}{ Repeatability $(n=6)$}} & \multirow{2}{*}{\multicolumn{2}{|c|}{ Stability $(24 \mathrm{~h}, n=6)$}} & \multirow{2}{*}{\multicolumn{2}{|c|}{ Recovery $(n=3)$}} \\
\hline & \multicolumn{2}{|c|}{ Intra-day } & \multicolumn{2}{|c|}{ Inter-day } & & & & & & \\
\hline & Mean (mg/g) & RSD (\%) & Mean $(\mathrm{mg} / \mathrm{g})$ & RSD (\%) & Mean (mg/g) & $\operatorname{RSD}(\%)$ & Mean $(\mathrm{mg} / \mathrm{g})$ & $\operatorname{RSD}(\%)$ & Recovery (\%) & RSD (\%) \\
\hline Gallic Acid & 0.41 & 0.93 & 0.40 & 1.07 & 0.80 & 1.81 & 0.53 & 1.73 & 100.22 & 3.03 \\
\hline Oxypaeoniflorin & 1.89 & 1.18 & 1.82 & 1.32 & 2.17 & 1.65 & 4.60 & 0.84 & 98.96 & 0.72 \\
\hline Paeonolide & 1.14 & 0.88 & 1.09 & 1.22 & 0.23 & 2.63 & 0.76 & 2.19 & 98.05 & 1.78 \\
\hline Paeoniflorin & 1.70 & 0.47 & 1.57 & 1.28 & 11.31 & 2.50 & 20.91 & 2.45 & 99.21 & 2.57 \\
\hline Benzoylpaeoniflorin & 1.12 & 0.64 & 1.04 & 2.08 & 1.29 & 1.78 & 1.15 & 1.98 & 98.84 & 1.18 \\
\hline Paeonol & 2.31 & 0.73 & 2.20 & 0.99 & 19.98 & 0.39 & 19.67 & 0.52 & 99.54 & 1.15 \\
\hline
\end{tabular}


Table 3. Contents of six analytes in Cortex Moutan of 50 batches $(\mathrm{mg} / \mathrm{g}, n=3)$.

\begin{tabular}{|c|c|c|c|c|c|c|}
\hline No. & Gallic acid & Oxypaeoniflorin & Paeonolide & Paeoniflorin & Benzoylpaeoniflorin & Paeonol \\
\hline $\mathrm{S} 1$ & $0.29 \pm 0.02$ & $3.07 \pm 0.13$ & $1.42 \pm 0.64$ & $25.40 \pm 0.44$ & $2.69 \pm 0.46$ & $25.44 \pm 0.43$ \\
\hline S2 & $1.10 \pm 0.17$ & $4.54 \pm 0.48$ & $0.71 \pm 0.31$ & $30.86 \pm 2.20$ & $5.79 \pm 0.09$ & $38.85 \pm 1.90$ \\
\hline S3 & $0.28 \pm 0.00$ & $3.03 \pm 0.06$ & $2.63 \pm 0.47$ & $23.67 \pm 2.16$ & $2.54 \pm 0.03$ & $22.68 \pm 1.51$ \\
\hline $\mathrm{S} 4$ & $0.19 \pm 0.01$ & $3.22 \pm 0.19$ & $2.52 \pm 0.37$ & $24.36 \pm 2.45$ & $2.58 \pm 0.13$ & $28.10 \pm 1.63$ \\
\hline S5 & $0.71 \pm 0.02$ & $3.11 \pm 0.02$ & $0.35 \pm 0.17$ & $16.99 \pm 1.27$ & $1.85 \pm 0.01$ & $19.50 \pm 0.04$ \\
\hline S6 & $0.33 \pm 0.02$ & $3.84 \pm 0.17$ & $0.28 \pm 0.11$ & $24.16 \pm 1.66$ & $2.58 \pm 0.02$ & $24.63 \pm 0.65$ \\
\hline S7 & $0.61 \pm 0.02$ & $5.48 \pm 0.28$ & $0.64 \pm 0.23$ & $27.65 \pm 1.25$ & $2.06 \pm 0.02$ & $34.51 \pm 0.07$ \\
\hline S8 & $0.90 \pm 0.03$ & $3.27 \pm 0.37$ & $0.18 \pm 0.09$ & $25.03 \pm 2.75$ & $2.67 \pm 0.16$ & $21.21 \pm 0.01$ \\
\hline S9 & $0.26 \pm 0.11$ & $4.74 \pm 0.56$ & $0.78 \pm 0.17$ & $24.47 \pm 1.23$ & $2.33 \pm 0.16$ & $22.95 \pm 1.01$ \\
\hline S10 & $0.13 \pm 0.02$ & $3.09 \pm 0.54$ & $0.31 \pm 0.04$ & $16.61 \pm 0.21$ & $2.12 \pm 0.16$ & $19.54 \pm 0.12$ \\
\hline S11 & $0.21 \pm 0.01$ & $4.34 \pm 0.17$ & $1.51 \pm 0.44$ & $21.88 \pm 0.39$ & $3.04 \pm 0.52$ & $23.69 \pm 0.21$ \\
\hline S12 & $0.29 \pm 0.02$ & $5.01 \pm 0.23$ & $0.93 \pm 0.37$ & $18.99 \pm 0.59$ & $1.51 \pm 0.15$ & $27.43 \pm 1.16$ \\
\hline S13 & $0.15 \pm 0.02$ & $3.05 \pm 0.21$ & $0.24 \pm 0.12$ & $13.87 \pm 0.97$ & $1.76 \pm 0.09$ & $19.17 \pm 1.33$ \\
\hline S14 & $0.20 \pm 0.00$ & $3.43 \pm 0.18$ & $1.33 \pm 0.02$ & $18.34 \pm 1.15$ & $1.86 \pm 0.09$ & $20.14 \pm 2.16$ \\
\hline S15 & $0.15 \pm 0.01$ & $3.31 \pm 0.10$ & $2.54 \pm 0.05$ & $19.04 \pm 0.90$ & $1.97 \pm 0.05$ & $16.53 \pm 1.23$ \\
\hline S16 & $0.19 \pm 0.01$ & $3.89 \pm 0.07$ & $2.51 \pm 0.06$ & $20.66 \pm 0.98$ & $2.33 \pm 0.03$ & $19.36 \pm 0.24$ \\
\hline S17 & $0.14 \pm 0.02$ & $4.16 \pm 0.34$ & $5.01 \pm 1.46$ & $16.23 \pm 1.06$ & $1.57 \pm 0.05$ & $15.87 \pm 0.33$ \\
\hline S18 & $0.42 \pm 0.04$ & $5.79 \pm 0.14$ & $9.02 \pm 0.54$ & $28.45 \pm 0.21$ & $2.45 \pm 0.40$ & $21.30 \pm 0.32$ \\
\hline S19 & $0.29 \pm 0.01$ & $8.80 \pm 0.34$ & $4.82 \pm 0.36$ & $30.93 \pm 1.28$ & $1.24 \pm 0.16$ & $27.58 \pm 1.30$ \\
\hline S20 & $0.16 \pm 0.02$ & $5.36 \pm 0.04$ & $4.70 \pm 0.94$ & $18.68 \pm 0.29$ & $1.19 \pm 0.11$ & $22.10 \pm 0.37$ \\
\hline S21 & $1.45 \pm 0.16$ & $2.74 \pm 0.34$ & $1.74 \pm 0.03$ & $16.34 \pm 2.04$ & $1.03 \pm 0.17$ & $19.56 \pm 1.19$ \\
\hline S22 & $1.54 \pm 0.41$ & $3.12 \pm 0.06$ & $2.83 \pm 0.19$ & $21.07 \pm 1.01$ & $1.35 \pm 0.42$ & $20.99 \pm 2.47$ \\
\hline S23 & $1.28 \pm 0.09$ & $3.26 \pm 0.01$ & $3.39 \pm 0.95$ & $23.54 \pm 0.89$ & $1.91 \pm 0.13$ & $21.75 \pm 1.76$ \\
\hline S24 & $0.47 \pm 0.02$ & $2.74 \pm 0.74$ & $0.73 \pm 0.30$ & $12.68 \pm 2.32$ & $1.18 \pm 0.11$ & $18.57 \pm 1.58$ \\
\hline S25 & $1.05 \pm 0.16$ & $2.65 \pm 0.27$ & $1.31 \pm 0.77$ & $16.95 \pm 0.45$ & $1.58 \pm 0.06$ & $19.25 \pm 2.97$ \\
\hline S26 & $0.92 \pm 0.05$ & $2.50 \pm 0.03$ & $2.81 \pm 0.25$ & $13.87 \pm 0.65$ & $0.48 \pm 0.05$ & $16.50 \pm 0.95$ \\
\hline S27 & $0.98 \pm 0.41$ & $2.58 \pm 0.12$ & $3.88 \pm 0.73$ & $16.27 \pm 1.56$ & $0.93 \pm 0.33$ & $20.71 \pm 0.21$ \\
\hline S28 & $0.80 \pm 0.05$ & $2.47 \pm 0.09$ & $3.65 \pm 0.58$ & $15.59 \pm 0.45$ & $0.85 \pm 0.01$ & $21.06 \pm 0.56$ \\
\hline S29 & $0.74 \pm 0.02$ & $3.34 \pm 0.17$ & $5.05 \pm 0.60$ & $15.44 \pm 1.51$ & $0.74 \pm 0.02$ & $18.20 \pm 0.32$ \\
\hline S30 & $0.69 \pm 0.01$ & $4.17 \pm 1.53$ & $1.82 \pm 0.10$ & $19.57 \pm 0.66$ & $1.85 \pm 0.22$ & $26.16 \pm 1.32$ \\
\hline S31 & $0.91 \pm 0.03$ & $2.05 \pm 0.35$ & $1.90 \pm 1.08$ & $11.24 \pm 0.25$ & $1.04 \pm 0.17$ & $19.93 \pm 1.28$ \\
\hline S32 & $1.06 \pm 0.00$ & $3.05 \pm 0.33$ & $3.54 \pm 0.17$ & $22.41 \pm 0.02$ & $2.09 \pm 0.06$ & $19.64 \pm 0.47$ \\
\hline S33 & $1.84 \pm 0.00$ & $2.17 \pm 0.16$ & $0.09 \pm 0.00$ & $10.49 \pm 0.55$ & $2.65 \pm 0.02$ & $17.78 \pm 2.50$ \\
\hline S34 & $2.07 \pm 0.06$ & $2.43 \pm 0.08$ & $0.03 \pm 0.03$ & $10.70 \pm 0.19$ & $1.67 \pm 0.05$ & $17.52 \pm 1.99$ \\
\hline S35 & $2.13 \pm 0.04$ & $1.85 \pm 0.05$ & $0.01 \pm 0.01$ & $7.33 \pm 0.17$ & $1.02 \pm 0.01$ & $20.17 \pm 2.77$ \\
\hline S36 & $1.08 \pm 0.10$ & $2.49 \pm 0.05$ & $0.12 \pm 0.08$ & $10.37 \pm 0.02$ & $1.93 \pm 0.02$ & $22.60 \pm 1.06$ \\
\hline
\end{tabular}




\section{Continued}

\begin{tabular}{|c|c|c|c|c|c|c|}
\hline S37 & $1.55 \pm 0.21$ & $1.37 \pm 0.21$ & $1.50 \pm 0.36$ & $8.94 \pm 1.65$ & $2.20 \pm 0.31$ & $16.45 \pm 1.69$ \\
\hline $\mathrm{S} 38$ & $0.51 \pm 0.54$ & $1.86 \pm 0.52$ & $0.55 \pm 1.60$ & $10.93 \pm 0.94$ & $1.87 \pm 0.80$ & $19.89 \pm 0.25$ \\
\hline $\mathrm{S} 40$ & $0.36 \pm 0.94$ & $3.08 \pm 0.41$ & $4.06 \pm 1.31$ & $11.48 \pm 0.87$ & $0.83 \pm 1.21$ & $18.48 \pm 0.21$ \\
\hline $\mathrm{S} 41$ & $0.22 \pm 0.01$ & $2.91 \pm 0.02$ & $2.07 \pm 0.43$ & $20.19 \pm 1.19$ & $1.24 \pm 0.52$ & $27.30 \pm 1.68$ \\
\hline $\mathrm{S} 42$ & $0.54 \pm 0.14$ & $2.99 \pm 0.36$ & $2.19 \pm 0.39$ & $13.33 \pm 1.02$ & $1.56 \pm 0.08$ & $18.21 \pm 0.53$ \\
\hline $\mathrm{S} 43$ & $0.63 \pm 0.08$ & $3.59 \pm 0.17$ & $5.01 \pm 0.89$ & $11.60 \pm 1.84$ & $1.25 \pm 0.29$ & $22.15 \pm 2.14$ \\
\hline S44 & $0.42 \pm 0.12$ & $4.03 \pm 1.47$ & $14.02 \pm 1.13$ & $15.54 \pm 0.71$ & $1.20 \pm 0.40$ & $13.58 \pm 0.68$ \\
\hline $\mathrm{S} 45$ & $0.22 \pm 0.12$ & $5.22 \pm 1.72$ & $4.19 \pm 0.11$ & $15.94 \pm 0.88$ & $0.97 \pm 0.09$ & $22.54 \pm 1.15$ \\
\hline S47 & $0.75 \pm 0.07$ & $3.66 \pm 0.22$ & $5.87 \pm 0.35$ & $12.50 \pm 0.25$ & $1.82 \pm 0.24$ & $18.34 \pm 1.56$ \\
\hline $\mathrm{S} 48$ & $0.90 \pm 0.09$ & $2.64 \pm 0.09$ & $3.02 \pm 0.28$ & $12.35 \pm 0.66$ & $1.60 \pm 0.11$ & $18.94 \pm 0.98$ \\
\hline S49 & $0.32 \pm 0.03$ & $3.88 \pm 1.16$ & $6.23 \pm 2.18$ & $10.95 \pm 1.92$ & $1.08 \pm 0.42$ & $16.55 \pm 1.75$ \\
\hline S50 & $0.25 \pm 0.04$ & $6.32 \pm 0.58$ & $10.45 \pm 0.50$ & $19.26 \pm 0.12$ & $2.92 \pm 0.59$ & $17.76 \pm 0.63$ \\
\hline
\end{tabular}

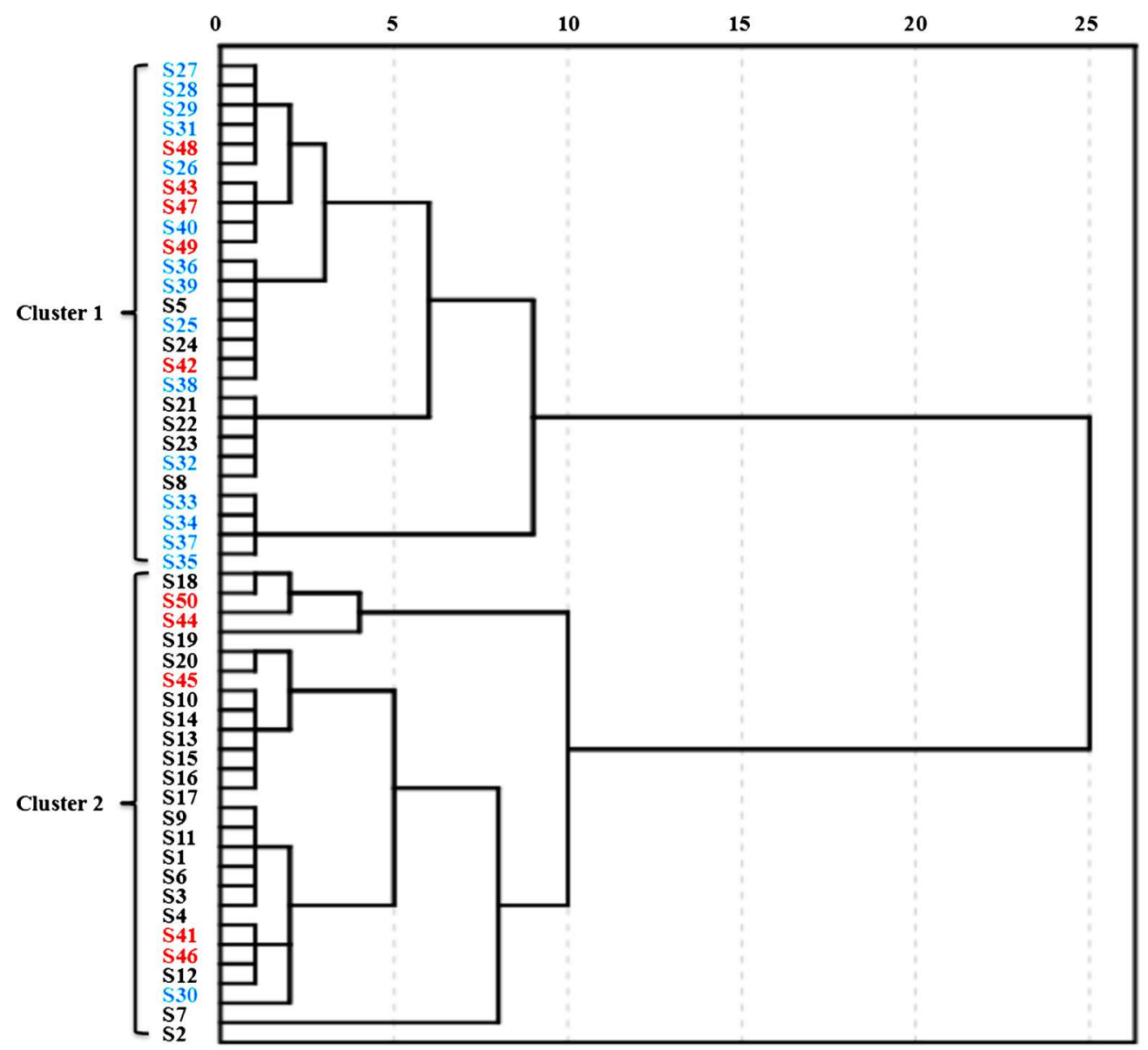

Figure 3. Hierarchical cluster dendritic diagram of 50 Cortex Moutan based on the content of six components different colors represent samples from different sources: S1 - S24: P. suffruticosa, S25 - S40: P. ostii, and S41 - S50: cultivars of P. suffruticosa. 
were generated by HCA. There were 26 samples including 15 samples sourced from $P$. ostii, six samples originated from $P$. suffruticosa and five samples planted from cultivars were formed the Cluster 1 , and another 24 samples were gathered into Cluster 2, including 18 samples sourced from P. suffruticosa, one sample from $P$. ostii and another five samples from cultivars. The HCA results indicated that most samples sourced from $P$. suffruticosa and samples sourced from $P$. ostii could be included in one branch, respectively. Surprisingly, $P$. ostii samples from Chongqing and Anhui province could be gathered into one group without affecting by geographical origins. While samples originated in cultivars were separated into other two clusters, which indicated instable chemical quality of CM from cultivars of $P$. suffruticosa. The present results revealed that the sources other than planting areas were the key factor for the quality stability of CM.

As CM originated from P. suffruticosa and P. ostii are the main materials of $\mathrm{CM}$ and they are also widely circulated on herbal market, the present study could discriminate the two sources by quantification of six compound markers. The established analytical method is effective for quality evaluation of CM.

\subsection{Measurement of Intestinal Absorption of Paeonol and Paeoniflorin}

Paeonol and paeoniflorin were chosen for intestinal absorption assay of $\mathrm{CM}$, as the two components accounted for $60 \%-90 \%$ amounts of the detected six components. The intestinal absorbed samples were analyzed using the quantitative method established in 2.4. The intestinal absorbed amounts of two ingredients were detected increasing with absorption time. Paeoniflorin showed higher intestinal absorption than that of paeonol in the two different parts of intestine (as shown in Table 4).

The absorption rates were then calculated and varied from $6.69 \%$ to $15.93 \%$

Table 4. The absorbed amounts of paeonol and paeoniflorin from CM extract.

\begin{tabular}{cccc}
\hline \multirow{2}{*}{ Absorption time (h) } & Component & \multicolumn{2}{c}{ Absorption $(\mathrm{mg} / \mathrm{g})$} \\
\cline { 3 - 4 } & & Jejunumpart $(\boldsymbol{n}=3)$ & Ileum part $(\boldsymbol{n}=3)$ \\
\hline \multirow{2}{*}{0.5} & Paeonol & $1.349 \pm 0.180$ & $1.595 \pm 0.068$ \\
& Paeoniflorin & $3.353 \pm 0.0647$ & $3.126 \pm 0.112$ \\
& Paeonol & $2.520 \pm 0.094$ & $1.692 \pm 0.081$ \\
1.0 & Paeoniflorin & $3.721 \pm 0.059$ & $3.598 \pm 0.103$ \\
& Paeonol & $2.456 \pm 0.730$ & $2.401 \pm 0.560$ \\
& Paeoniflorin & $4.891 \pm 0.079$ & $4.211 \pm 0.839$ \\
& Paeonol & $2.849 \pm 0.087$ & $3.214 \pm 0.455$ \\
& Paeoniflorin & $5.041 \pm 0.103$ & $4.553 \pm 0.364$ \\
\hline
\end{tabular}

Values are presented as mean $\pm \mathrm{SD}, n=3$. Samples were collected from jejunum and ileum at $0.5,1.0,1.5$ and $2.0 \mathrm{~h}$ after incubation with $\mathrm{CM}$ extract. 

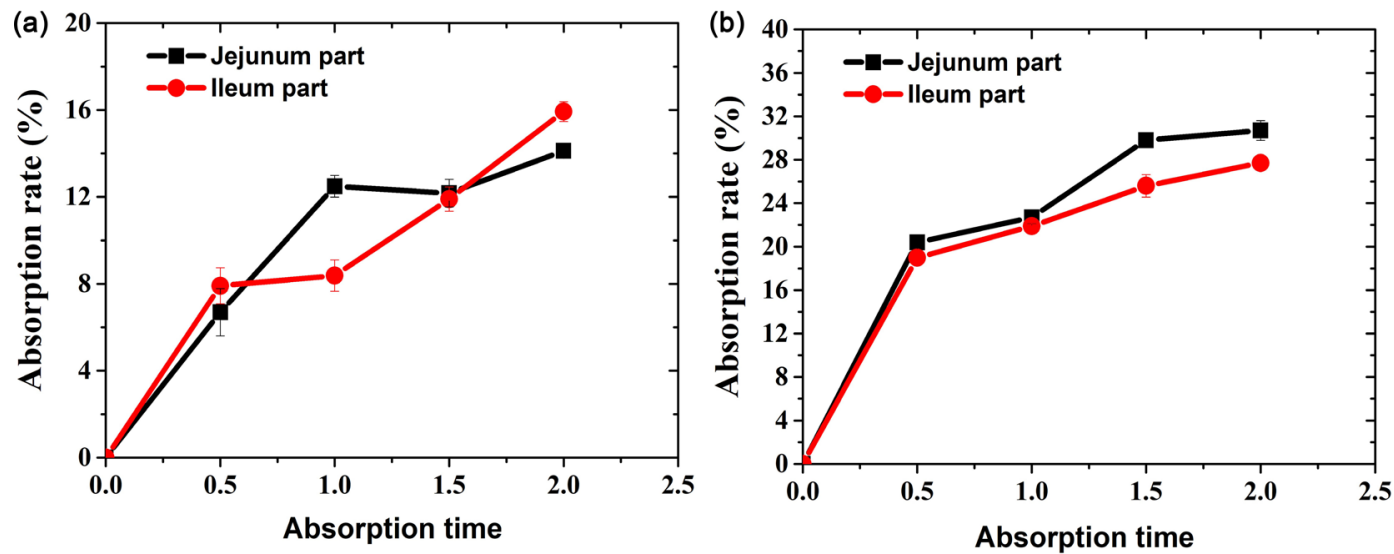

Figure 4. Graphical representation of the absorption rates of paeonol and paeoniflorin with time. Absorption rate $=$ Absorption amounts of component/amounts of incubation component, $n=3$.

for paeonol in different absorption time. The absorption rates were found similar in jejunum and ileum at $0.5,1.5$ and $2.0 \mathrm{~h}$, except at $1.0 \mathrm{~h}$, in which paeonol had higher absorption rate in jejunum than that in ileum (Figure 4(a)). For paeoniflorin, the absorption rate showed great difference in jejunum and ileum with the range of $19 \%-30.70 \%$ at different absorption time. Figure 4(b) showed paeoniflorin had similar absorption activities in jejunum and ileum.

The intestinal absorption test indicated that paeoniflorin and paeonol could be absorbed in jejunum and ileum, and the absorbed amounts increased with time. paeoniflorin showed higher absorption rates than that of paeonol.

\section{Conclusion}

The findings obtained from multi-component quantification of CM clearly indicated that $\mathrm{CM}$ originated from $P$. suffruticosa and $P$. ostii showed different chemical properties, and that the two materials had related stable quality, while CM sourced from cultivars of $P$. suffruticosa showed great variety on chemical quality. The outcomes from intestinal absorption test in vitro specified that paeoniflorin and paeonol presented different absorption characteristics, in which paeoniflorin was detected having higher absorption rate than that of paeonol. However, further research is needed to elucidate the real pharmacological effects of these absorbed components for pharmacological evaluation of CM. The experimental findings suggest that the established chemical method could be taken as a powerful tool for the quality evaluation of CM from different sources. Meanwhile, the intestinal absorption properties of CM could provide useful information for further pharmacological evaluation of CM.

\section{Acknowledgements}

This work was financially supported by Chongqing Research Program of Basic Research and Frontier Technology (cstc2016jcyjA0359), the Fundamental Research Funds for the Central Universities (XDJK2016C073, SWU115043) and the National Natural Science Foundation of China (81703644). 


\section{Conflicts of Interest}

The authors declare no conflicts of interest regarding the publication of this paper.

\section{References}

[1] Zhang, Y.X., Yu, D., Liu, C.Y. and Gai, S.P. (2018) Dynamic of Carbohydrate Metabolism and the Related Genes Highlights PPP Pathway Activation during Chilling Induced bud Dormancy Release in tree Peony (Paeonia suffruticosa). Scientia Horticulturae, 242, 36-43. https://doi.org/10.1016/j.scienta.2018.07.022

[2] Chinese Pharmacopeia Commission (2015) Pharmacopoeia of the People's Republic of China. Vol. 1, 172.

[3] Peng, H.S., Wang, D.Q., Peng, D.Y. and Huang, L.Q. (2017) Research and Investigation on Original Plants of Medicinal Moutan. China Journal of Chinese Material Medica, 42, 1632-1636.

[4] Qiu, Y.X, Huang, J.H., Jiang, X.M., Chen, Y., Liu, Y., Zeng, R., Shehla, N., Liu, Q., Liao, D.F., Guo, D., Liang, Y.Z. and Wang, W. (2015) Quantitative and Qualitative Determination of LiuweiDihuang Preparations by Ultra High Performance Liquid Chromatography in Dual-Wavelength Fingerprinting Mode and Random Forest. China Journal of Chinese Materia Medica, 38, 3720-3726.

[5] Wang, S.Y., Huang, J., Mao, H.J., Wang, Y.L., Kasimu, R., Wei, X. and Wang, J.H. (2014) A Novel Method HPLC-DAD Analysis of the Contents of Moutan Cortexand Paeoniae Radix Alba with Similar Constituents-Monoterpene Glycosides in Guizhi Fuling Wan. Molecules, 19, 17957-17967.

https://doi.org/10.3390/molecules191117957

[6] Luo, S., Wen, R.Y., Wang, Q., Zhao, Z.X., Nong, F.F., Fu, Y.J., Huang, S.W., Chen, J.Y., Zhou, L. and Luo, X. (2019) Rhubarb Peony Decoction Ameliorates Ulcerative Colitis in Mice by Regulating Gut Microbiota to Restoring Th17/Treg Balance. Journal of Ethnopharmacology, 231, 39-49. https://doi.org/10.1016/j.jep.2018.08.033

[7] Guo, B.L., Basang, D.J., Xiao, P.G. and Hong, D.Y. (2002) Research on the Quality of Original Plants and Material Medicine of Cortex Paeoniae. China Journal of Chinese Material Medica, 27, 654-657.

[8] Li, L.Z.; Song, N. and Wang, X.L. (2014) Studies on Mechanism of Cortex Moutan on Removing Heat to Cool and Promoting Blood Circulation to Diminish Carbuncle. Chinese Archives of Traditional Chinese Medicine, 32, 863-865.

[9] Ding, L.Q., Jiang, Z.H., Liu, Y., Chen, L.X., Zhao, Q., Yao, X.S., Zhao, F. and Qiu, F. (2012) Monoterpenoid Inhibitors of NO Production from Paeonia suffruticosa. Fitoterapia, 83, 1598-1603. https://doi.org/10.1016/j.fitote.2012.09.008

[10] Ding, L.Q., Zhao, F., Chen, L.X., Jiang, Z.H., Liu, Y., Li, Z.M., Qiu, F. and Yao, X.S. (2012) New Monoterpene Glycosides from Paeonia suffruticosa Andrews and Their Inhibition on NO Production in LPS-Induced RAW 264.7 Cells. Bioorganic \& Medicinal Chemistry Letters, 22, 7243-7247. https://doi.org/10.1016/j.bmcl.2012.09.034

[11] Zhao, D.D., Jiang, L.L., Li, H.Y., Yan, P.F. and Zhang, Y.L. (2016) Chemical Components and Pharmacological Activities of Terpene Natural Products from the Genus Paeonia. Molecules, 21, pii: E1362.

[12] Ding, L.Q., Zuo, Q.F., Li, D.D., Feng, X.C., Gao, X.M., Zhao, F. and Qiu, F. (2017) A New Phenone from the Roots of Paeonia suffruticosa Andrews. Natural Product Research, 31, 253-260. https://doi.org/10.1080/14786419.2016.1230114 
[13] Wang, Z.Q., He, C.N., Peng, Y., Chen, F.H. and Xiao, P.G. (2017) Origins, Phytochemistry, Pharmacology, Analytical Methods and Safety of Cortex Moutan (Paeonia suffruticosa Andrew): A Systematic Review. Molecules, 22, pii: E946.

[14] Zhang, M.H., Feng, L., Zhu, M.M., Gu, J.F., Jiang, J., Cheng, X.D., Ding, S.M., Wu, C. and Jia, X.B. (2014) The Anti-Inflammation Effect of Moutan Cortex on Advanced Glycation End Products-Induced Rat Mesangial Cells Dysfunction and High-GlucoseFat Diet and Streptozotocin-Induced Diabetic Nephropathy Rats. Journal of Ethnopharmacology, 151, 591-600. https://doi.org/10.1016/j.jep.2013.11.015

[15] Lu, L., Qin, Y.T., Chen, C. and Guo, X.M. (2018) Beneficial Effects Exerted by Paeonol in the Management of Atherosclerosis. Oxidative Medicine and Cellular Longevity, 2018, 1-11. https://doi.org/10.1155/2018/1098617

[16] Jiao, J.J., Sun, L.L., Guo, Z.Y., Hou, S., Holyst, R., Lu, Y. and Feng, X.Z.(2016) Antibacterial and Anticancer PDMS Surface for Mammalian Cell Growth Using the Chinese Herb Extract Paeonol(4-Methoxy-2-Hydroxyacetophenone). Scientific Reports, 6, Article No. 38973.

[17] Zhang, J.M., Wang, J.X., Xia, T. and Zhou, S.L. (2009) DNA Barcoding: Species Delimitation in Tree Peonies. Science in China Series C: Life Sciences, 52, 568-578. https://doi.org/10.1007/s11427-009-0069-5

[18] Ma, H., Tang, S.W., Zhang, J. and Zhang, L.X. (2011) Study on the Effect of Processing Methods and the Storage Period on Quality Factors of Moutan Cortex. Research and Practice on Chinese Medicines, 25, 16-17.

[19] Deng, A.P., Fang, W.T., Xie, D.M., Jin, Y., Zhao, Y.Y., Peng, D.Y., Zhan, Z.L. and Guo, L.P. (2017) Transition of Producing Areas and Quality Evaluation of Mudanpi in Different Period of History. Modern Chinese Medicine, 19, 880-890.

[20] Yan, Y., Zhang, Q.Q. and Feng, F. (2016) HPLC-TOF-MS and HPLC-MS/MS Combined with Multivariate Analysis for the Characterization and Discrimination of Phenolic Profiles in Nonfumigated and Sulfur-Fumigated Rhubarb. Journal of Separation Science, 39, 2667-2677. https://doi.org/10.1002/jssc.201501382

[21] Guo, L., Zeng, S.L., Zhang, Y., Li, P. and Liu, E.H. (2016) Comparative Analysis of Steroidal Saponins in Four Dioscoreae Herbs by High Performance Liquid Chromatography Coupled with Mass Spectrometry. Journal of Pharmaceutical and Biomedical Analysis, 117, 91-98. https://doi.org/10.1016/j.jpba.2015.08.038

[22] Hou, X.H., Ge, M., Zhang, Y.C. and Zeng, Z.L. (2018) HPLC Fingerprint Study and Cluster Analysis of Paeonia suffruticosa. China Pharmacy, 29, 3354-3358.

[23] Tambe, A., Mokashi, P. and Pandita, N. (2019) EX-Vivo Intestinal Absorption Study of Boswellic Acid, Cyclodextrin Complexes and Poloxamer Solid Dispersions Using Everted Gut Sac Technique. Journal of Pharmaceutical and Biomedical Analysis, 167, 66-73. https://doi.org/10.1016/j.jpba.2018.12.018

[24] Utami, R.A., Hakiki, A., Asyarie, S. and Retnoningrum, D.S. (2018) Gliadin Peptide Facilitates FITC Dextran Transport across the Non Everted Gut Sac of Rat Small Intestine. Scientia Pharmaceutica, 86, 13.

https://doi.org/10.3390/scipharm86020013 